\title{
The role of functional phosphoric acid ester monomers in the surface treatment of yttria-stabilized tetragonal zirconia polycrystals
}

\author{
Masami ARAI, Tomohiro TAKAGAKI, Akifumi TAKAHASHI and Junji TAGAMI \\ Cariology and Operative Dentistry, Department of Oral Health Sciences, Graduate School of Medical and Dental Sciences, Tokyo Medical and Dental \\ University, 1-5-45, Yushima, Bunkyo-ku, Tokyo 113-8510, Japan \\ Corresponding author, Tomohiro TAKAGAKI; E-mail: takagaki.ope@tmd.ac.jp
}

\begin{abstract}
This study aimed to evaluate the bond strength of a resin cement to yttria-stabilized tetragonal zirconia polycrystals (Y-TZPs) treated with three different phosphate functional monomers containing primers by measuring the water contact angle. Seventy Y-TZP discs were divided into seven experimental groups $(n=10)$, including an untreated (control) group. Specimens were treated with one of the following monomers: MDP (MD), GPDM (GP), and Phenyl-P (PP). After primer application and air drying, half the treated specimens were ultrasonically cleaned in ethanol for $2 \mathrm{~min}$ (MD-e, GP-e, and PP-e), and contact angles were measured. The tensile bond strength test was performed after storage for $24 \mathrm{~h}$ in $37^{\circ} \mathrm{C}$ water. The contact angle on the MD-e surface $\left(64^{\circ}\right)$ was significantly higher than that on the Con, GP-e, and PP-e surfaces $\left(51^{\circ}-52^{\circ}\right)$. The application of the MDP-containing primer resulted in significantly higher bond strength between Y-TZP ceramics and a resin cement.
\end{abstract}

Keywords: Phosphoric functional monomer, Yttria-stabilized tetragonal zirconia, Tensile bond strength, Contact angle, Adhesion

\section{INTRODUCTION}

In recent years, yttria-stabilized tetragonal zirconia polycrystal (Y-TZP) ceramics have become increasingly popular in dentistry due to their superior optical properties, biocompatibility, and mechanical properties than other conventional all-ceramic materials ${ }^{11}$. Y-TZP ceramics have been used as a metal-free alternative for conventional dental materials for various dental clinical applications such as abutments for implants, crowns, and fixed partial prostheses ${ }^{2}$. However, a significant drawback of these ceramics is their limited ability to adhere to resin cements because of their glass-free polycrystalline microstructure ${ }^{3)}$. Moreover, traditional silane coupling agents used with silica-based ceramics are ineffective with zirconia ${ }^{4)}$ due to the lack of silica in Y-TZP ceramics.

To overcome this problem, several methods that improve the bonding performance between resin cement and Y-TZP ceramics have been reported. Particle air abrasion using $\mathrm{Al}_{2} \mathrm{O}_{3}$ has been reported to provide effective initial bond strength through micromechanical interlocking, although spontaneous failure may occur following storage for a period of time $\mathrm{e}^{5,6}$. A tribochemical silica coating (Rocatec and Cojet, 3M ESPE, St. Paul, MN, USA) can be created by abrasion with airborne silicacoated aluminum particles that result in the formation of a silica layer on the roughened Y-TZP surfaces due to high-speed impact ${ }^{7,8}$. This silica layer then allows silanization with a silane coupling agent ( $\gamma$-MPTS) to produce a chemical bonding, which significantly enhances the bond strength between a treated substrate and resin cement ${ }^{9)}$. However, several studies have also

Color figures can be viewed in the online issue, which is available at J-STAGE.

Received Feb 9, 2016: Accepted Oct 3, 2016

doi:10.4012/dmj.2016-060 JOI JST.JSTAGE/dmj/2016-060 reported that water storage and thermal cycling result in a decrease in the bond strength between silica-coated Y-TZP surfaces and resin cements ${ }^{10)}$.

Recently, adhesive strategies that combine chemical and mechanical pre-treatment have been developed. Kern and Wegner ${ }^{7)}$ reported that sandblasting with $\mathrm{Al}_{2} \mathrm{O}_{3}$ and resin cements containing 10-methacryloyloxydecyl dihydrogenphosphate (MDP) as a monomer is essential to achieve strong and durable bonding to zirconia ceramics. Therefore, air abrasion at $0.2-0.3 \mathrm{MPa}$ combined with MDP-containing primer/cement is the most popular method for achievement of a strong and reliable bond between Y-TZP and resin-based cements ${ }^{11,12)}$. However, there are a limited number of studies that focus on the effects of acidic phosphate monomers on the Y-TZP surface after the application.

Therefore, the purpose of this study was to measure the water contact angle formed on a Y-TZP ceramic surface and to examine the role of functional phosphoric acid ester monomers on the bond strength of a resin cement.

\section{MATERIALS AND METHODS}

Experiment 1 -Contact angle measurement

The materials used are listed in Table 1. Seventy Y-TZP discs (Lava, 3M ESPE) were fabricated according to the manufacturer's instructions. The surfaces of the specimens were wet-polished using \#600-grid SiC paper and was then cleaned with deionized water for $5 \mathrm{~min}$ and $99 \%$ ethanol for $2 \mathrm{~min}$. They were then randomly divided into seven experimental groups (Fig. 1), including one control (left untreated; Con group). The experimental specimens were treated with three different phosphate functional monomers [MDP (MD), 
Table 1 Composition of the materials used in this study

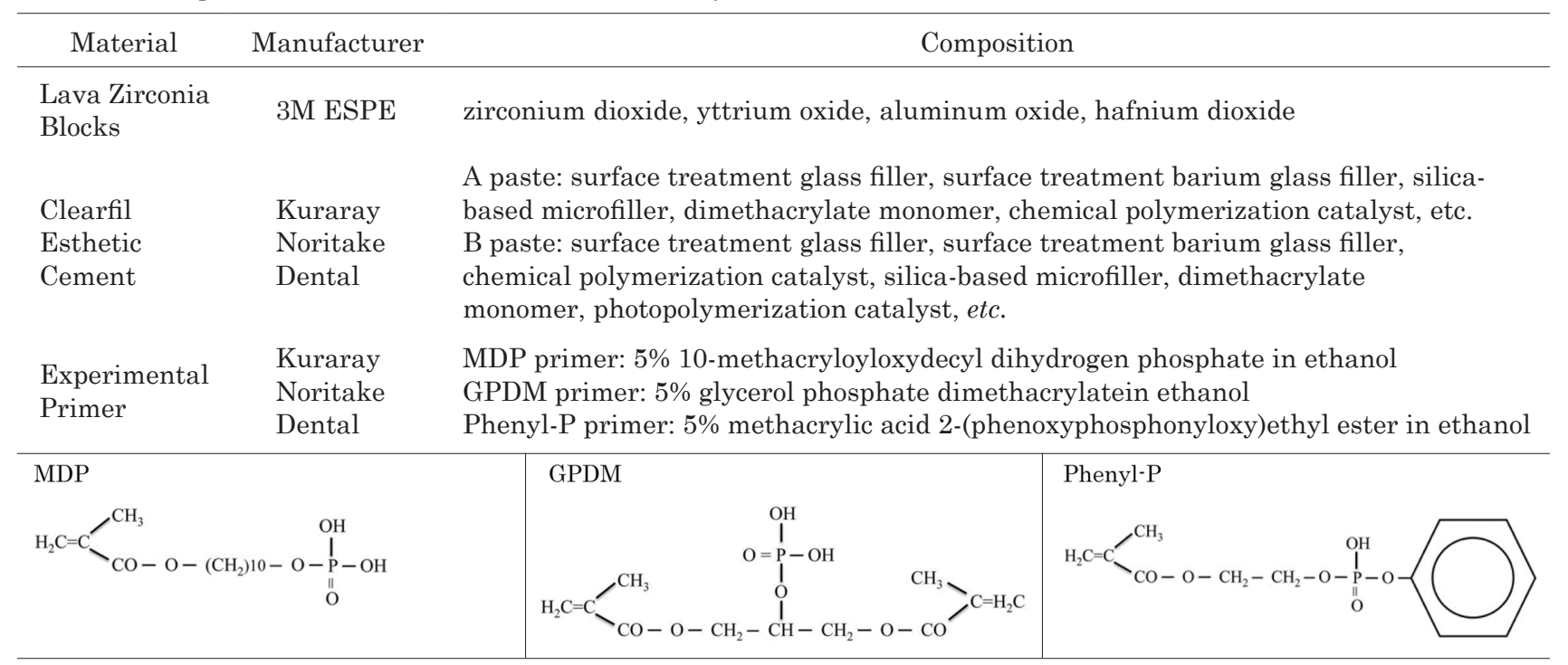

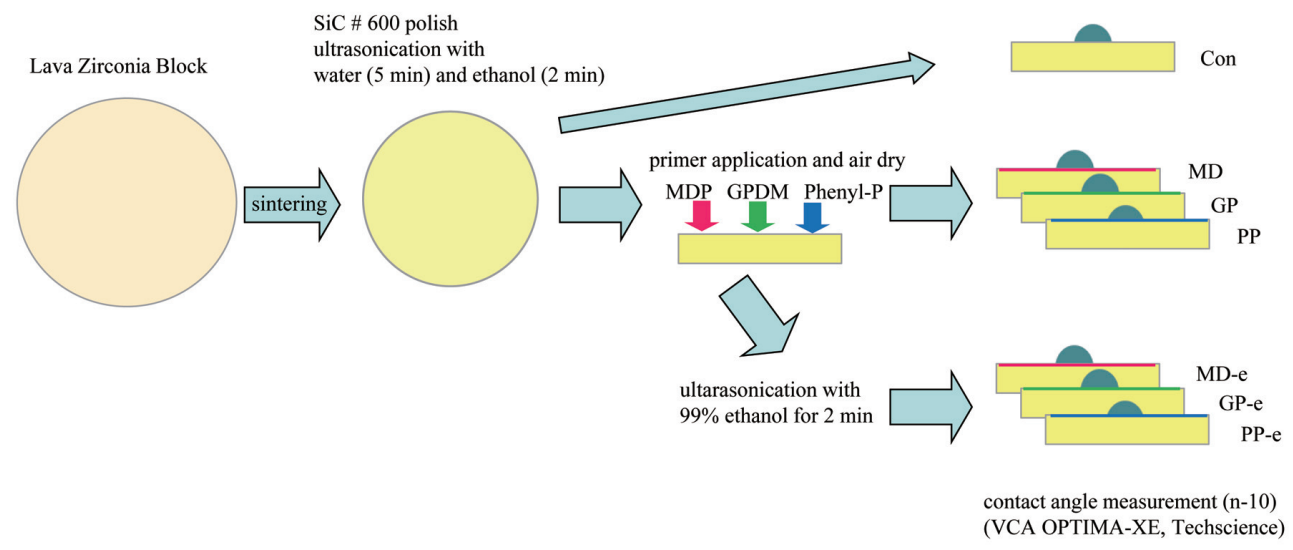

Fig. 1 Schematic illustration of the methodology of specimen preparation for contact angle measurement.

GPDM (GP), and Phenyl-P (PP)] containing primers (experimental primer, $5 \%$ in ethanol, Kuraray Noritake Dental, Tokyo, Japan) for $10 \mathrm{~s}$. After primer application and air drying with forceful air, half the specimens treated with the primers were ultrasonically cleaned in ethanol for $2 \mathrm{~min}$ to remove unreacted functional monomers (MD-e, GP-e, and PP-e). Thereafter, a deionized water droplet was placed on the surface of each specimen $(n=10)$, and the contact angle was measured using a contact angle measuring device (VCA OPTIMAXE, Techscience, Koshigaya, Japan). Each measurement was repeated at least three times, and the mean value was reported. Data were statistically analyzed using Dunnett's T3 test with Bonferroni correction and 95\% confidence intervals.

\section{Experiment 2-Tensile bond strength test}

Seventy Y-TZP discs were prepared in the same manner as mentioned above, sandblasted with aluminum oxide (50 $\mu \mathrm{m}, 0.2 \mathrm{MPa}, 10 \mathrm{~s}, 10 \mathrm{~mm}$ distance), and cleaned with deionized water for $5 \mathrm{~min}$ and ethanol for $2 \mathrm{~min}$ (Fig. 2). After surface cleaning with $40 \%$ phosphoric acid gel (K-etchant, Kuraray Noritake Dental), the specimens were randomly divided into seven experimental groups (Con group or pre-treated with the three different phosphate functional monomers containing primers mentioned above) for $10 \mathrm{~s}$. After primer application and air drying with forceful air, half the primer treated specimens were ultrasonically cleaned in ethanol for 2 min to remove unreacted functional monomers (MDe, GP-e, and PP-e). A piece of aluminum tape with a circular hole of $4 \mathrm{~mm}$ diameter and $100 \mu \mathrm{m}$ thickness was placed on the specimen surface to demarcate the 


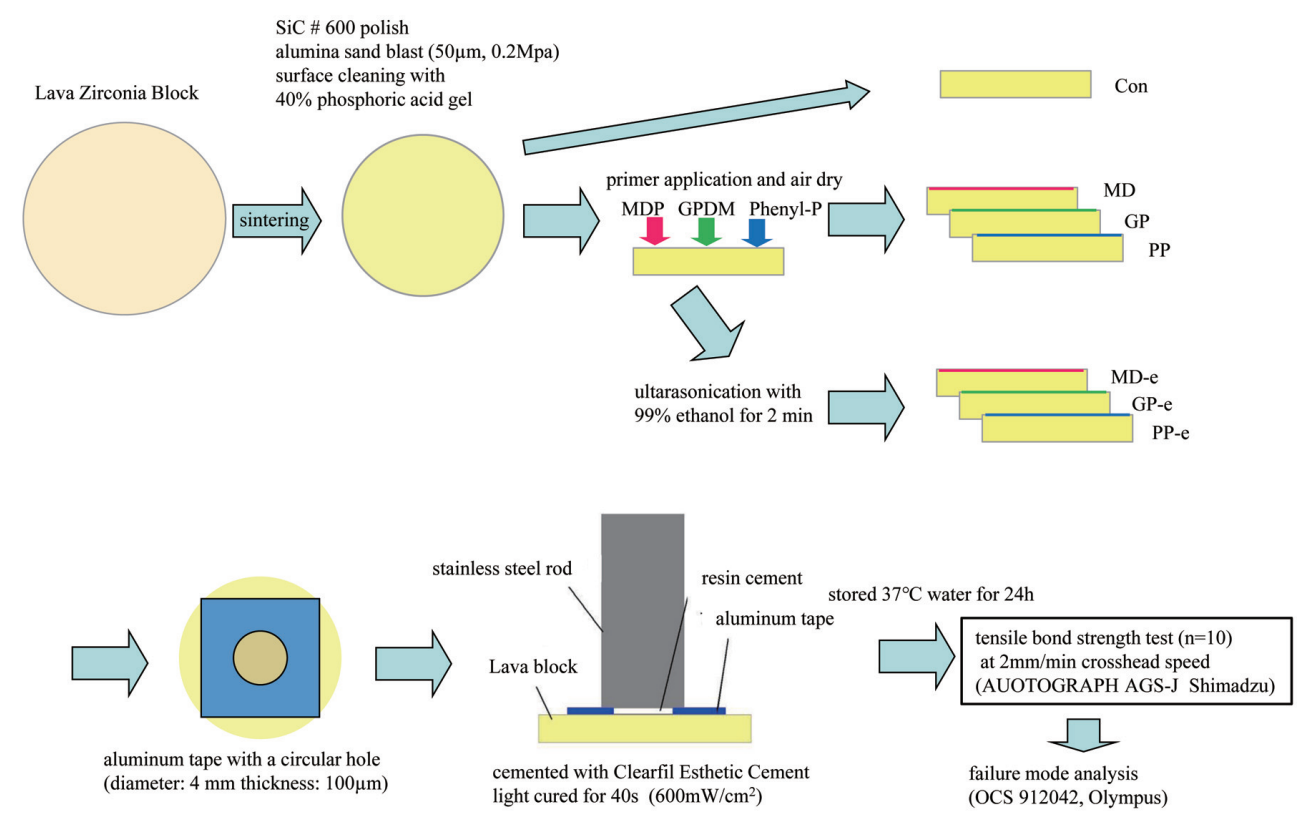

Fig. 2 Schematic illustration of the methodology of specimen preparation for the tensile bond strength test.

Table 2 Means and standard deviations of contact angles $\left(^{\circ}\right)$ of all groups $(n=10)$

\begin{tabular}{ccccc}
\hline & MD & GP & PP & Con \\
\hline Ethanol cleaning (-) & n.d. ${ }^{*}$ & $24.2 \pm 5.6$ & $33.6 \pm 2.7$ & $51.2 \pm 3.2^{\text {a }}$ \\
Ethanol cleaning (+) & $65.4 \pm 3.5$ & $54.9 \pm 5.0^{\text {a }}$ & $49.9 \pm 4.0^{\text {a }}$ & - \\
\hline
\end{tabular}

Means followed by the same superscript letter are not statistically different $(p>0.05)$.

* not detected due to water droplet spread out

bonding area. An MDP-free dual-cured resin cement (Clearfil Esthetic Cement, Kuraray Noritake Dental) was applied to the treated surface as a handle with the help of a metal primer-treated stainless steel rod for the subsequent tensile bond testing and was then light cured with a halogen light (Optilux 501, $600 \mathrm{~mW} / \mathrm{cm}^{2}$, Demetron, Danbury, CT, USA) for $40 \mathrm{~s}$. The specimens were stored at room temperature for $1 \mathrm{~h}$, followed by water at $37^{\circ} \mathrm{C}$ for $24 \mathrm{~h}$. Tensile bond strengths were measured using a universal testing machine (Autograph AGS-J, Shimadzu, Kyoto, Japan) at a crosshead speed of $2 \mathrm{~mm} / \mathrm{min}$. Data were statistically analyzed using Dunnett's T3 test with Bonferroni correction and 95\% confidence intervals. After tensile bond strength testing, the failure mode was examined under a light microscope (OCS 912042, Olympus, Tokyo, Japan). Failure modes were classified into the following two categories: adhesive failure between ceramics and resin cements and mixed failure including cohesive failure within resin cements. Data were analyzed using the chi-square test with Bonferroni correction and 95\% confidence intervals.

\section{RESULTS}

\section{Experiment 1 -Contact angle measurement}

The contact angle on the MD surface could not be measured due to the spreading of the water droplet. Table 2 shows the contact angles of each group. The GP and PP groups exhibited significantly lower contact angles than the Con group $(p<0.05)$; however, these differences ceased to exist after ethanol ultrasonic cleaning $(p>0.05)$. The contact angle on the MD-e surface $\left(65.4^{\circ}\right)$ was significantly higher than that of the Con, GP-e, or PP-e groups.

\section{Experiment 2-Tensile bond strength test}

The results of the tensile bond strength test are summarized in Table 3. MD and MD-e exhibited the highest tensile bond strengths among the tested groups, being significantly higher than that of the PP, PP-e, and Con groups $(p<0.05)$. Ethanol ultrasonic cleaning after primer application tended to improve the tensile bond strength of each monomer, but these differences were not 
Table 3 Means and standard deviations of tensile bond strength (MPa) for all groups $(n=10)$

\begin{tabular}{ccccc}
\hline & MD & GP & PP & Con \\
\hline Ethanol cleaning (-) & $12.5 \pm 3.9^{\mathrm{a}}$ & $9.1 \pm 3.8^{\mathrm{ac}}$ & $4.9 \pm 1.6^{\mathrm{bc}}$ & $4.5^{\mathrm{a}} 1.2^{\mathrm{bc}}$ \\
Ethanol cleaning (+) & $13.8 \pm 3.8^{\mathrm{a}}$ & $11.7 \pm 4.8^{\mathrm{ac}}$ & $7.2 \pm 2.7^{\mathrm{bc}}$ & - \\
\hline
\end{tabular}

Means followed by the same superscript letters are not significantly different $(p>0.05)$.

Table 4 Failure mode distribution in all groups (adhesive failure/cohesive and mixed failure, $n=10$ )

\begin{tabular}{lllll}
\hline & MD & GP & PP & Con \\
\hline Ethanol cleaning (-) & $0 / 10^{*}$ & $10 / 0$ & $10 / 0$ & $10 / 0$ \\
Ethanol cleaning (+) & $5 / 5$ & $10 / 0$ & $10 / 0$ & - \\
\hline
\end{tabular}

* represents a statistically significant difference in distribution $(p<0.05)$ as seen in chi-square test.

statistically significant. The failure mode distribution is summarized in Table 4. All specimens, except MD and MD-e, exhibited adhesive failure between the ceramic and resin cement. Half the MD-e and all MD specimens exhibited cohesive or mixed failure within the resin cement. The chi-square test indicated that MD $(p<0.05)$ was significantly different.

\section{DISCUSSION}

To achieve durable bonding with zirconia-based ceramics, surface cleaning to remove contamination (oil, saliva protein, etc.), surface roughening to achieve mechanical interlocking, and improvement of surface wettability using phosphate functional monomers are essential before the application of resin cements. Dental resin cements that do not contain any phosphate functional monomers exhibit hydrophobic characteristics due to the nature of their monomers. The wettability of luting cements is one of the factors that predicts adhesive properties between resin cements and adherends. The monolayer chemically bonded phosphate functional monomers create relatively low surface energy in zirconia ceramics, leading to a higher contact angle ${ }^{13)}$. Therefore, measuring the contact angle of a water droplet serves as a convenient indicator of the surface modification of zirconia ceramics with phosphate functional monomers.

In this study, the contact angle of the Con group was $51.2^{\circ}$, and this was in agreement with a previous study that used the same material surface ${ }^{14)}\left(52.7^{\circ}\right)$. MD-e exhibited a significantly higher contact angle than the GP-e, PP-e, and Con groups and significantly higher tensile bond strength than the PP-e and Con groups. MDP is one of the most frequently used phosphate functional monomers and was originally designed to enhance bond strength to tooth substrate and non-precious metal alloys. Similar to the interaction of phosphoric functional monomers with hydroxyapatite ${ }^{15,16)}$, a chemical interaction is believed to occur between the functional monomer and the hydroxyl group of metal ions on the ceramic surface ${ }^{17)}$. Kern et al. ${ }^{18)}$ reported that a combination of alumina particle air abrasion and priming with MDP-containing primers provided durable, long-term bonding to dental zirconia ceramics. MD exhibited the highest bond strength among all treated specimens, regardless of whether the primer-treated surface had undergone ethanol cleaning. These adhesive monomers are believed to possess the ability to chemically adsorb onto metal oxide surfaces, resulting in secondary forces such as van der Waals forces or hydrogen bonds at the resin-zirconia interface ${ }^{19)}$. These adsorbed monomers are arranged in a single layer with the hydrophobic methacryloyloxy group on the surface, resulting in an improvement in the surface wettability of zirconia ceramic surfaces to resin cements.

The groups that did not undergo ethanol ultrasonic cleaning after primer application exhibited lower contact angles, regardless of the choice of phosphoric functional monomer. Ethanol evaporates form the primer on the ceramic surface while air dry, unreacted monomer must have accumulated and left unevenly on the surface. Phosphate functional monomers possess both hydrophilic and hydrophobic characteristics, and the initial dentin bond strength is influenced by the length and hydrophilicity of their spacer chains ${ }^{20)}$. Thus, in the absence of ethanol cleaning, the hydrophilic parts of the phosphoric functional monomers were scattered on the surface, resulting in lower contact angles than those of the Con group.

Ethanol cleaning resulted in the contact angles of GP-e and PP-e significantly differing from that of the Con group, and they also exhibited higher tensile bond strength. Chemical interactions of GPDM and Phenyl-P with zirconia ceramic surfaces do not exhibit ordered arrangement due to steric hindrance, resulting in adhesive failure between the ceramic and resin 
cement. Cohesive failure within the resin cement was observed in the MD and MD-e groups, with a better bonding performance, despite the fact that they exhibited completely different surface characteristics. The balanced molecular structure of MDP makes its soluble in water and dimethacrylate monomers ${ }^{20)}$. In the MD group, MDP accumulated after primer application and air drying may have dissolved into and polymerized with the dimethacrylate monomers in the resin cement. The degree of conversion of a resin can be affected by the functional monomer ${ }^{21}$. The degree of conversion of resin cement may have been affected by contamination with accumulated MDP left on the surface in the MD group which, along with improved adhesion, may have resulted in the higher frequency of cohesive failure observed in this group. These results suggest that the molecular structure of phosphoric functional monomers strongly influences the chemical interaction and bonding performance of resin cements with zirconia ceramics. Further investigations focusing on long-term bond durability and the mechanism of chemical interaction between zirconia ceramics and functional monomers are necessary.

\section{CONCLUSIONS}

Within the limitations of this study, the following conclusions were drawn:

a) MDP-containing primers exhibited the best bonding performance among all tested phosphoric functional monomers and b) their chemical interaction ability was strongly influenced by the molecular structure.

\section{ACKNOWLEDGMENTS}

This research was supported by the Japan Society for the Promotion of Science (No.24792016).

\section{REFERENCES}

1) Denry I, Kelly JR. State of the art of zirconia for dental applications. Dent Mater 2008; 24: 299-307.

2) Tinschert J, Natt G, Mautsch W, Augthun M, Spiekermann H. Fracture resistance of lithium disilicate-, alumina-, and zirconia-based three-unit fixed partial dentures: a laboratory study. Int J Prosthodont 2001; 14: 231-238.

3) Valandro LF, Ozcan M, Amaral R, Leite FP, Bottino MA. Microtensile bond strength of a resin cement to silica-coated and silanized In-Ceram Zirconia before and after aging. Int $J$ Prosthodont 2007; 20: 70-72.

4) Aboushelib MN, Kleverlaan CJ, Feilzer AJ. Microtensile bond strength of different components of core veneered all-ceramic restorations. Part 3: double veneer technique. J Prosthodont 2008; 17: 9-13.

5) Wegner SM, Kern M. Long-term resin bond strength to zirconia ceramic. J Adhes Dent 2000; 2: 139-147.

6) Wolfart M, Lehmann F, Wolfart S, Kern M. Durability of the resin bond strength to zirconia ceramic after using different surface conditioning methods. Dent Mater 2007; 23: 45-50.

7) Kern M, Wegner SM. Bonding to zirconia ceramic: adhesion methods and their durability. Dent Mater 1998; 14: 64-71.

8) Tanaka R, Fujishima A, Shibata Y, Manabe A, Miyazaki T. Cooperation of phosphate monomer and silica modification on zirconia. J Dent Res 2008; 87: 666-670.

9) Chen L, Suh BI, Kim J, Tay FR. Evaluation of silica-coating techniques for zirconia bonding. Am J Dent 2011; 24: 79-84.

10) Akgungor G, Sen D, Aydin M. Influence of different surface treatments on the short-term bond strength and durability between a zirconia post and a composite resin core material. J Prosthet Dent 2008; 99: 388-399.

11) Inokoshi M, Kameyama A, De Munck J, Minakuchi S, Van Meerbeek B. Durable bonding to mechanically and/or chemically pre-treated dental zirconia. J Dent 2013; 41: 170179.

12) Yang B, Barloi A, Kern M. Influence of air-abrasion on zirconia ceramic bonding using an adhesive composite resin. Dent Mater 2010; 26: 44-50.

13) Chen L, Suh BI, Brown D, Chen X. Bonding of primed zirconia ceramics: evidence of chemical bonding and improved bond strengths. Am J Dent 2012; 25: 103-108.

14) Kim MJ, Kim YK, Kim KH, Kwon TY. Shear bond strengths of various luting cements to zirconia ceramic: surface chemical aspects. J Dent 2011; 39: 795-803.

15) Yoshida Y, Nagakane K, Fukuda R, Nakayama Y, Okazaki M, Shintani H, Inoue S, Tagawa Y, Suzuki K, De Munck J, Van Meerbeek B. Comparative study on adhesive performance of functional monomers. J Dent Res 2004; 83: 454-458.

16) Fukegawa D, Hayakawa S, Yoshida Y, Suzuki K, Osaka A, Van Meerbeek B. Chemical interaction of phosphoric acid ester with hydroxyapatite. J Dent Res 2006; 85: 941-944.

17) Ikemura K, Endo T. A review of the development of radical photopolymerization initiators used for designing light-curing dental adhesives and resin composites. Dent Mater J 2010; 29: 481-501.

18) Kern M, Barloi A, Yang B. Surface conditioning influences zirconia ceramic bonding. J Dent Res 2009; 88: 817-822.

19) Feitosa VP, Ogliari FA, Van Meerbeek B, Watson TF, Yoshihara K, Ogliari AO, Sinhoreti MA, Correr AB, Cama G, Sauro S. Can the hydrophilicity of functional monomers affect chemical interaction? J Dent Res 2014; 93: 201-206.

20) Feitosa VP, Sauro S, Ogliari FA, Ogliari AO, Yoshihara $\mathrm{K}$, Zanchi CH, Correr-Sobrinho L, Sinhoreti MA, Correr AB, Watson TF, Van Meerbeek B. Impact of hydrophilicity and length of spacer chains on the bonding of functional monomers. Dent Mater 2014; 30: e317-e323.

21) Oguri M, Yoshida Y, Yoshihara K, Miyauchi T, Nakamura Y, Shimoda S, Hanabusa M, Momoi Y, Van Meerbeek B. Effects of functional monomers and photo-initiators on the degree of conversion of a dental adhesive. Acta Biomater 2012; 8: 19281934. 